\title{
Energy Balancing Algorithms in Wireless Sensor Networks
}

\author{
Anne-Lena Kampen \\ Bergen University College, Bergen \\ ITEM NTNU, Trondheim \\ Norway \\ Anne-Lena.Kampen@hib.no \\ anneleka@stud.ntnu.no
}

\author{
Knut Øvsthus \\ Bergen University College, Bergen \\ Norway \\ knut.ovsthus@hib.no
}

\author{
Øivind Kure \\ NTNU, Trondheim \\ Norway \\ okure@item.ntnu
}

\begin{abstract}
The energy consumption in Wireless Sensor Networks, WSN, need to be balanced in order to avoid early depletion of nodes. In this paper we use a common context to analyze a broad range of the energy balancing algorithms suggested in literature. In addition we suggest three new algorithms to complete the range. Altogether, nine different balancing techniques are analyzed. We focuses on networks running the IPv6 Routing Protocol for Low-Power and Lossy Networks (RPL) routing protocol. Our simple change in RPL's parent selection procedure can give a significant balancing effect without any increase in management cost. However, the best balancing algorithm is when the nodes exchange residual-energy information to ensure forwarding through the highest residual-energy nexthop node. The increased information exchange implies increased management cost due to the amount of information transmitted and added computational load.
\end{abstract}

\section{INTRODUCTION}

$\mathrm{W}$ IRELESS sensor networks (WSN) generally consist of wireless nodes with a collective objective of gathering measured information at the sink [1]. The monitored area may be large compared to the nodes' transmission range. Hence, the information needs to be relayed to reach the sink. The topology of the relaying paths may create imbalance in the traffic share, and therefore the energy consumption, between the nodes. Energy imbalance results in lifespan variation between the nodes. Observations of real networks in [2] and [3] show that some nodes relay a substantial portion of the traffic, thus they become hot-spot-nodes having a high energy consumption rate.

Depleted or dead nodes make the gathered data incomplete and, more important they may cause network partitioning. Applying energy balancing routing algorithms levels the traffic load, hence lifetime, between the nodes. The ideal situation is long living WSNs where all nodes have equal lifetime. However, this ideal situation not feasible due to the increased traffic density toward the sink in multihop networks. The goal in multihop networks is instead to balance the energy consumption between nodes at equal hop distance from the sink. Network management will be simplified if the nodes at each rank have similar lifetime. Balancing algorithms are the topic of this paper.
Our contribution is threefold. First we present a methodical review of a broad range of energy balancing algorithms. The algorithms range from approaches requiring simple changes of the applied routing algorithms, to approaches that require complex add-ons. Second we use a common context to compare these algorithms. Third, we suggest three new balancing algorithms to complete the collection of balancing algorithms found in the literature. The tree suggested algorithms are random selection of preferred-parent, conserving of Single Point of Failure (SPOF) parent and energy balancing based on eavesdropping.

To get a good estimate of the energy pattern in the network we use the nodes residual-energy. The residual-energy gives the true picture of the energy variation that appears between the nodes. In addition, residual-energy is directly related to the nodes lifetime.

To evaluate the impact of different energy balancing techniques, we use the routing protocol suggested by Internet Engineering Task Force (IETF) for use in WSN, IPv6 Routing Protocol for Low-Power and Lossy Networks (RPL) [4]. RPL creates routing entries in the nodes which forms an overall destination oriented directed acyclic graph (DODAG) rooted at the sink. The graph is created by broadcasting of DODAG Information Object (DIO) messages. The sink initiates the transmission, and the messages are further broadcasted throughout the whole network. The DIO includes the senders' rank information. The rank indicates a node's distance to the sink. The rank increases as the distance to the sink increases. The sink is at rank 0 and the sink's one-hop neighbor defines the rank-one nodes and so forth. Each node caches a parent-list containing all neighbors that report a rank equal to the lowest rank heard. A preferred-parent is selected among the nodes in the parent-list. The preferredparent is used as the current next-hop node on the path toward the sink. To maintain the DODAG, the nodes transmit DIO messages periodically at intervals decided by a trickle timer [5].

The rest of the paper is structured as follows. In Section 2 we present related work, in Section 3 introduces the different energy balancing approaches to be analyzed, the 
simulation is presented in Section 4, and Section 5 comprises the conclusion.

\section{RELATED WORK}

Several energy balancing approaches are suggested in the literature. Energy balancing based on selecting the most energy optimal path is suggested in [6] - [13]. In all but the two latter of these algorithms are energy information exchanged through DIO messages. Applying DIO messages to exchange energy information means the energy balancing depends on the number of data packet transmitted per transmitted DIO message. Thus, increased energy balance is paid by increased DIO transmission frequency, which means increased average energy consumption. Further, the trickle timer [5] decides the DIO emission frequency such that the emission frequency decreases exponentially with time in converged networks. Hence, the balancing effect of the algorithms discussed in the next paragraph will decline with time.

The object function (OF) for RPL suggested in [6] defines the path cost as the energy level of the node on the path with lowest residual-energy. The node that advertises the highest path cost is preferred as selected parent, and the lower energy nodes are spared. The authors of [8] suggest that the node with the highest remaining energy among the nodes with the lowest expected transmission count (ETX) is chosen as the preferred-parent in network running RPL. Both ETX and node energy is used to select between parent nodes of equal hop-count in [9]. The residual-energy is included as a denominator in the additive distance metric in [10]. Using it as in the denominator makes the cost of a node increase toward infinity as energy approaches zero. Hence, the paths including low energy nodes is avoided due to their high cost. A routing metric that calculate the expected lifetime of the nodes is defined in [11]. The expected lifetime is calculated as the ratio of the node's residual-energy over the total energy spent to transmit data. The paths including the most constrained nodes are avoided by defining the path weight as the minimum expected lifetime along the path. An approach similar to RPL is used to create paths for networks with multiple sinks in [7]. Several equal-rank nodes are cached as potential parent based primary on hop-count metric, secondary the nodes energy metric and third on the highest link-quality-indication. Algorithms where the highest residual-energy path is selected or the lowest residual-energy paths are avoided are part of our analysis.

Energy information is exchange through the ACK packet in the approach presented in [13]. The network run RPL, and the nodes perform a weighted selection to choose among its available next-hop nodes. The selection is weighted between distributing the traffic through the lowest delay path and distributing the traffic to nodes with higher remaining energy. In the routing protocol suggested in [12], the energy information is both piggybacked on data packets and included in the ACK packets.
Hop-count is used as the metric to generate parent-list. Data packets are transmitted to the highest energy member of the parent-list. If there is no parent node available, the packet is transmitted to the sibling node with the highest amount of energy. Our analysis includes an algorithm where the ACK message exchange the energy information.

Energy consumption can be balanced by continuously spreading the transmitted data over multiple paths, and such methods are also part of our analysis. Approaches using multiple paths are suggested in [14] [15]. RPL is used as the routing protocol in [14] where the forwarding load is weighted between the members of the parent-list. The weighting is based on the members' residual-energy. The transmission range dynamically adjusted to maintain $\mathrm{k}$ parents. Energy information is exchanged between the nodes through ACK and DIO packet. In addition is also hello packets mentioned as possible information carriers. The approach in [15] enables multipath data forwarding through energy-sufficient paths, as opposed to minimumenergy-cost paths. They propose a routing algorithm which makes a hierarchical routing graph similar to RPL. The nodes forward packets through alternate paths to extend the network lifetime. The conditions of the paths are monitored by the sink which re-initiate path search if the number of working paths gets lower than two. Multiple paths are also discussed in the surveys presented in [16] [17]. Survey [16] cites an algorithm presented in [18], which takes both the energy level and hop distance into account to allocate different data rates to multiple disjoint paths. The sink decides the rate of the different paths and assign messages are sent form the sink to the source nodes to inform about the path rates. The top-down survey paper [17] cites an interesting improved cost function used to balance the energy consumption among the nodes [19]. The improved cost calculation algorithm makes the cost increase rapidly with decrease in the nodes remaining energy. Hence, traffic is directed away from hot-spot nodes. The approach requires that the nodes cache several states for each neighbor and that energy information is exchanged periodically. The survey paper [17] also discusses energy balancing by using a few relay nodes with enhanced capabilities. In addition they discuss use of mobile sinks. These algorithms increase the start-up management cost of the networks, and increases the network cost.

Clustering is among the energy efficient algorithm discussed the survey presented in [20] and suggested to improve energy utilization in [26]. The basic idea of energy efficient clustering is to perform energy efficient rotation of the clusterhead assignment and let the clusterhead perform energy efficient management of the local cluster traffic. Clustering is not part of our analysis as it is not very well fitted for RPL running network.

Balancing the energy consumption by making the nodes alternate between direct transmissions to the sink and using multi-hop transmissions is suggested in [21]. 
The protocol is used as an extension to RPL in [22] which presents a smart/green test-bed of nodes spanning across several smart offices. The findings of [22] shows that the protocol suggested in [21] balances the network energy consumption compared to classic RPL. However, it is more energy expensive giving an overall increased energy dissipation. This algorithm is only considered for one-hop networks, while we are considering multihop networks.

\section{BALANCING NETWORK ENERGY CONSUMPTION}

In this section we present the different energy balancing algorithms that are analyzed. Our hypothesis is that introducing small changes in the parent selection procedure improves the WSN energy balance, while substantial enhancements come at a cost of increased management complexity and information exchange between nodes. Further, efficient energy balance is achieved when focusing on reducing the load of the hot-spot nodes.

The following text lists nine algorithms. The tree new algorithms that we suggest are A: Randomize parent selection, D: Weighting round-robin based on SPOF-parent energy level and $\mathrm{H}$ : weighting round-robin based on eavesdropping.

\section{A. Randomize parent selection}

As a first approach to enhance the energy balance in WSN, we suggest a simple change in the preferred-parent selection algorithm. The aim of the suggested algorithm is to reduce the probability of creating hot-spot nodes. The probability is reduced by preventing that several child nodes select the same preferred-parent if other potential parents exist. According to the RPL algorithm, all nodes cache a parent-list containing all candidate parent nodes. A preferred-parent are selected among the parent-list nodes, using a specific parameter as tiebreaker. Hence, nodes with globally good tiebreaker value will be selected by all potential child nodes and may therefore become hot-spot nodes.

Our suggested algorithm creates a small change in the preferred-parent selection procedure to reduce the probability of creating such hot-spot nodes. The nodes randomly select a preferred-parent among the nodes in the parent-list instead of using a preordain tiebreaker parameter value. Hence, the probability that several potential child nodes select the same node as preferred-parent is reduced. The forwarding load is therefore more balanced. The weakness of the algorithm is that it can give energy consumption imbalance if selected parents are located such that they represent single paths for other nodes.

\section{B. Round-robin through multiple paths}

Selecting a single preferred-parent may overload some potential parents while leaving some potential parents unused. Thus, our analysis comprises an approach where this imbalance is alleviated by making the nodes transmit data packets to all nodes in their parent-list in a round- robin fashion. The approach shares the forwarding load equally between all members of the nodes' parent-lists. The main weakness of the approach is the load imbalance that is created between nodes with different number of child nodes.

\section{Weighted round-robin based on energy information in DIO messages}

To level the energy imbalance that may appear using the round-robin approach we implement algorithms in which the nodes exchange energy information during DIO transmission. The information is used to perform a weighted-fair-sharing between the parent nodes. Thus, the nodes share the traffic load among the nodes in the parent-list according to their relative residual-energy level. The energy-balancing effect of the weighted algorithms depends on the freshness of the energy information cached for the nodes in the parent-list. Hence, increased DIO exchange frequency means improved energy balance. However, increased DIO exchange frequency increases the energy consumption in the network. Thus, there is a tradeoff between energy balance and average energy consumption.

Weighted round-robin ensures that the energy depletion rate of the low energy parents is reduced, hence the energy balance is improved. However, the algorithm preserves the existing energy imbalance relationship between parent nodes.

\section{Weighting round-robin based on SPOF-parent energy level}

The goal of our single point of failure (SPOF) algorithm is to prevent early depletion of SPOF nodes. We define SPOF nodes as nodes that are part of one or more parent-lists containing only one member. In other words, a child that has a SPOF parent is disconnected from the routing graph if the parent node dies. Child of SPOF parent forwards all data through the SPOF parent. Even when the SPOF parent has a very low energy level, the child has no other option than continue forwarding through the SPOF parent. Hence, depleting of the SPOF node is continued.

To reduce the depletion rate of the SPOF nodes we suggest to direct traffic originating from higher rank nodes away from the SPOF nodes. In order to do so, we let nodes with a SPOF parent advertise the energy level that is the lowest of its own and its SPOF-parent's residual-energy level. Thus, traffic is directed away from the paths including the SPOF node.

Directing the traffic away from the SPOF nodes may come at an expense of other low energy nodes on the same rank as the SPOF node. However, child with SPOF parent continue to transmit their own generated data to their SPOF parent, while other nodes only get a weighted amount of traffic from their respective child nodes.

The DIO is used to exchange energy information. 


\section{E. Weighted round-robin based on prediction parents energy consumption}

The energy information gained through received DIO can be used to predict the energy consumption pattern in between DIO updates. To test such energy prediction algorithms, we implement an algorithm that estimate parents current energy level based on statistics of former energy consumption. The algorithm is as follows. The residual-energy a node advertises in consecutive transmitted DIOs is cached at the receiving nodes. The timespan between the consecutive DIO is further used to estimate the depletion rate of the transmitting node. The current energy level is estimated using the individual parents' energy drain rate and last advertised energy level. The estimated energy level is used to perform weighted-fair-sharing between the parent nodes.

\section{F. Weighted round-robin while avoid lowest energy parent}

In order to focus on the hot-spot nodes we suggest a partly weighted algorithm. The data is weighted between the parent nodes. However, no data is transmitted to the parent with the lowest residual-energy. Hence, the load on the hot-spot nodes is reduced. This algorithm requires that the nodes exchange residual-energy information through the DIO message.

\section{G. Use the highest energy parent node}

In the multiple path approaches, although weighted, each parent receives data for forwarding from their child nodes. This applies even if the residual-energy level of the parent is low. Hence, if all nodes transmit approximately equal amount of traffic, the nodes that are members of several paths are depleted faster than other nodes.

The depletion pace of low energy nodes is reduced in approaches where the lowest energy parent is avoided such as the approach presented in subsection III.F. However, nodes forces parents with second lowest energy level to forward traffic. Hence, the lowest energy nodes alternates their states with next lowest energy nodes.

A simple solution is to use only the highest energy parent node as the next-hop node. This algorithm is similar to the algorithm used in [8]. We implemented this algorithm and used DIO to exchange energy information.

\section{H. Weighting round-robin based on eavesdropping}

Utilizing information conveyed in DIOs may give an incomplete view of the current energy levels of the nodes in the parent-list. The reason is that traffic imbalance, and associated energy consumption imbalance that occurs between DIO transmissions are not taken into account.

In order to predict parents' energy consumption between DIO updates, we suggest that nodes eavesdrop on the traffic transmitted in the area. The algorithm operates as follows. Nodes read the source and destination address information in the eavesdropped traffic. The address is matched against the content in the parent-list of the eavesdropping nodes. When a match is found, the energy level of the associated parent-list entry is reduced according to the eavesdropped information. The energy level of the nodes in the parent-list is then used to perform weightedfair-sharing.

Eavesdropping does not significantly influence on the nodes energy consumption. The reason is that overheard packets destination address has to be read anyway to determine the intended receiver of the packet. The energy consumption due to overhearing is not taken into account when comparing the different balancing techniques. The reason is that the extent of overhearing energy consumption is mainly decided by the energy saving approach chosen at the MAC layer, while we are concentrating on the routing layer algorithms' impact on energy consumption.

The eavesdropped traffic may not give a complete overview of the parent nodes traffic load. For instance, child nodes of a common parent may not receive each other's packets due to hidden node. Thus, the calculations of the energy consumption of the parent-list nodes may be imprecise.

\section{Weighting round-robin based on energy information conveyed in ACK packets}

Lastly, we implement an algorithm in which information about the nodes' energy variation in between DIO transmissions is exchanged through ACK packets. ACK packets are sent as a response of received data packet. Hence, the nodes achieve a complete overview of the diverse energy levels of the nodes in their parent-list as each parent relays a packet.

However, the energy information of parents with low residual-energy is less current. The reason is that low energy nodes seldom forward data as they have low weight. In addition, nodes that rarely transmit data can have stale energy information for the nodes in their parent-lists. This may give temporary screwed forwarding load among parent nodes. However, the energy levels are continuously

TABLE 1.

Color-codes used in the figures to define the different energy balancing algorithms

\begin{tabular}{|l|}
\hline A Randomize parent \\
\hline B Round-robin \\
\hline C Weighted - DIO information \\
\hline D Weighted - SPOF parent \\
\hline E Weighted - predicted energy \\
\hline F Weighted - avoid lowest \\
\hline G Use highest energy node \\
\hline H Weighted - eavesdropping \\
\hline I Weighted -ACK information \\
\hline
\end{tabular}


balanced as energy information is updated, smoothing the discrepancy over time.

Weighted-fair-sharing is performed based on the energy information.

\section{iV. Simulations}

We perform simulations to evaluate the different discussed algorithms. The energy consumption in WSN increases toward the sink as the inner nodes are obligated to relay traffic for outer nodes. Thus, we mainly present simulations segregated on the node's rank. Applying an energy balancing algorithm will not change the average energy consumption for the nodes at the different rank since the total number of packet transmitted through each rank is unchanged.

We concentrate on transmitting and receiving energy consumption. Overhearing energy is not taken into account. The reason for omitting the overhearing energy consumption is that we concentrate on energy balancing at the network layer, and overhearing energy consumption is strongly dependent on the energy saving approach applied at the MAC layer. Overhearing may give a small variation in the average energy due to the chosen path. However, the difference between the energy consumed due to overhearing become negligible because the algorithms are compared at given average node density.

As discussed above, the average energy consumption at each rank is consistent regardless of applied balancing algorithm. However, an efficient energy balancing algorithm makes the residual-energy of the most depleted node approach the average residual-energy at the given rank. Hence, we present the average and minimum residual-energy after each node has generated 100 data packets.

Energy information is used to tune the traffic load between parent nodes in some of the evaluated algorithms. In networks running these algorithms, each node caches residual-energy information of its parents. The accuracy of the cached information depends on the update interval. For the algorithms that exchange energy information through DIO messages, the accuracy is improved by reducing the number data packets exchanged per DIO transmitted. The residual-energy information accuracy approaches the accuracy of the ACK algorithms if the DIO emission frequency approaches the data rate. However, increased DIO exchange frequency increases average energy consumption. Further, the DIO exchange frequency is decided by the trickle timer such that the exchange frequency is strongly reduced in converged stable networks. Thus, the energy balance is declining over time when the balance depends on DIO exchanged information.

In our initial simulations, 100 data packets create sufficient network traffic to discriminate the balancing effect of the different category of balancing algorithms. However, each node transmit a total of 16 DIO messages during the simulation runs, hence the number of data packets transmitted for each DIO transmission is low. Thus, to improve the basis of comparison we present additional simulation results for the weighting algorithms. Only two DIO messages are transmitted during the simulation run in these additional simulations, and the number of transmitted data packets is increased to 300 . This gives a more fair comparison between the algorithms relying on DIO to exchange energy information, relative to the algorithms that use additional means to exchange energy information.

We evaluate the different energy balancing approaches by performing simulations in OMNET++ [23], using the MiXiM module for wireless communication. The nodes' energy consumption is calculated based on traffic load. Based on the observations and references in [24], we assume that receiving and transmission of data packets consume the same amount of energy. The different types of packets have different packet sizes. Management packets are assumed to be half the size of data packets, while ACK packets are one tenth of the size of the data packets. These relative values are chosen based on an assumption that data packets never need the maximum allowed packet sizes as they are mainly limited to carry only measured data, while management packets only carries strictly needed information. Maximum data frame sizes and ACK frame sizes information extracted from 802.15.4 datasheet [25].

The nodes are randomly distributed in an $800 \mathrm{~m}$ times $800 \mathrm{~m}$ area. The nodes transmission range is $141 \mathrm{~m}$. The number of nodes is varied such that the node density changes from 8 to 20 . The node density is defined as the number on nodes inside a circle with radius equal to the nodes transmission range. Every simulation point presented represents the average value of 30 simulation runs with different seeds for random deployment of nodes.

As discussed above, the average energy consumption is equal for each rank over all energy balancing algorithms. However, the residual- energy values of the most depleted nodes change with the applied balancing algorithm. The most optimal energy balancing algorithm is the algorithm in which the residual-energy of the most depleted nodes converges to the average value. Therefore, we compare the algorithms with respect to their ability to make the nodes with lowest residual-energy approach the average value of their associated rank. Figure 1 shows simulation results that demonstrate to what extent the different algorithms make the lowest and average values converge. All the algorithms discussed in Section 3 are presented in the figure.

The nodes' average residual-energy, as well as the residual-energy of the most depleted nodes are presented in Figure 1. This is the residual-energy level of the nodes after each node has generated and transmitted 100 data packets. In addition to data, management traffic has been exchanged to make the network converge. Further has periodic DIO updates been transmitted. The circled shaped 

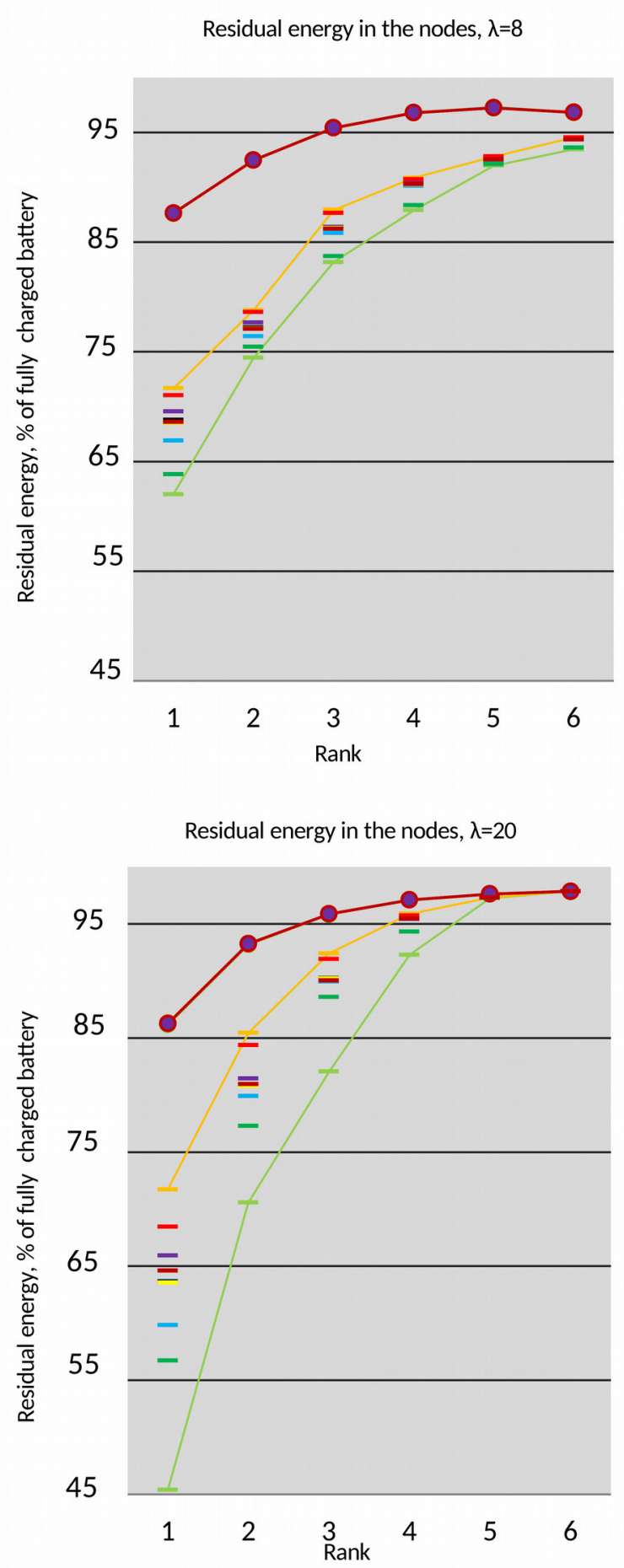

Fig 1. Residual-energy in the nodes after each node has generated 100 data packets.

markers with the corresponding lines show the average residual-energy values. The energy levels for the most depleted nodes are shown as short horizontal markers. In order to clarify the information displayed in the figure, the best and the worst of the residual-energy levels are displayed with solid curves through their associated markers.
Hence, the orange curve cut through the markers representing the highest residual-energy level and light green curve cut through the markers representing the lowest residual-energy. The colors of the markers and lines indicate the corresponding energy-balancing algorithm as defined in Table 1. To prevent that the important information gets hidden in an overloaded display, the $95 \%$ confidence interval is not shown in the figures. However, the $95 \%$ confidence interval is always within $7 \%$ of the average values. Simulations performed for node densities of $\lambda$ $=10$ and $\lambda=15$ show the same trends as shown for $\lambda=8$ and $\lambda=20$ in Figure 1.

The simulation results displayed in Figure 1 shows that native RPL creates energy imbalanced networks. The native RPL simulation results are represented by the light green marks and the light green curve. Native RPL gives the overall lowest residual-energy for all ranks and all node densities. The reason is that a fixed parent is used throughout the whole simulation scenario, further is lowest node-id used as a tiebreaker when choosing between potential parent nodes. The latter means that several nodes choose the same preferred-parent node.

The difference between the lowest residual-energy node and the average value increases toward the sink. The reason is the increased traffic density. A given imbalance in traffic share causes an increase in the real traffic load difference as the total traffic increases.

The energy imbalance increases rapidly with node density for approaches where the parent node is fixed. This is observed in Figure 1 where the light green native RPL line rapidly moves away from the average line as the node density increases. The reason is the increased number of neighbors. Increased number of neighbors increases the number of child nodes for the fixed parent.

Based on the discussion above it is clear that some kind of energy balancing techniques should be added to networks running native RPL. Our suggested random preferred-parent selection algorithm presented in subsection III.A, demands a minor change in the RPL implementation. Nevertheless, the residual-energy of the most depleted node is reduce by over $10 \%$ compared to native RPL for high density networks. This is seen in the Figure 1 comparing the light green native RPL marks with the dark green marks.

However, the most efficient energy balancing algorithm is the one presented in subsection III.G, in which the parent with the highest residual-energy is selected as the next-hop node. The algorithm is represented by the orange marks in the Figure 1. Using highest energy parent increases the residual-energy of the most depleted node more than $25 \%$ compared to the native RPL. The merit of the algorithm is that the residual-energy nodes are avoided. This result corresponds to the results presented in [8] and [6] where ETX is used as a metric to populate the parent-list. However, using the parent with the highest residual-energy means that energy information has to be 
exchanged between the nodes. Randomizing parent selection, presented in subsection III.A, does not add any overhead.

The weighted share algorithms increase the residualenergy of the most depleted nodes with 15 to $20 \%$ compared to native RPL in high density networks. The weighted share algorithms are presented in subsection III.C-F and III.H-I. The improved performance complies with the studies performed in in [14] [15]. However, the poorer performance of these algorithms compared to the highest energy parent algorithm, III.G, is due to the fact that the lowest energy parent is still used, although rarely.

In subsection III.F we suggest the improved weighting algorithm, where the most depleted node is avoided while the traffic is weighted between the other parents. The improved algorithm is represented by the red marks in Figure 1, and shows that the residual-energy of the most depleted node is always distinguishable higher than the general weighting algorithms.

The round-robin approach, represented by the light blue marks, contributes less to balance the energy than the weighting algorithms. The reason is that parent with low residual-energy are loaded with the same amount of traffic as the other parents.

The simulated weighted-fair-sharing algorithms exchange energy information through DIO messages as well as through the algorithm-specific energy information exchange technique. Thus, the energy information update intervals between the different algorithms converge if the DIO exchange frequency is high compared to the packet exchange frequency. This phenomenon is demonstrated in Figure 1 as it is difficult to discern between the simulation results for the algorithms that use weighting as balancing technique. To better illustrate the difference between the weighting algorithms we performed simulations where each node generated 300 data packets. The DIO exchange is limited such that each node only generates two DIOs during the whole simulation. The simulation result is shown in Figure 2.

As expected, when the DIO exchange frequency is reduced, the ACK method has an improved balancing performance relative to weighting based on DIO information. The ACK method is presented in subsection III.I and weighting based on DIO information is presented in subsection III.C. The improved balancing performance is seen in Figure 2 where the blue marker of the ACK method is closer to the average values than the yellow DIO information markers. In Figure 1, the blue ACK markers are actually hidden by the yellow markers. Hence, the ACK and the DIO information performed equally well when the number of data packets per DIO packet is low.

The eavesdrop-algorithm described in subsection III.H and represented with the black marks and line in the Figure 2 , has the worst balancing capabilities. This applies especially for the one-hop nodes. The reason is increased traffic density in these areas of the network. High traffic density means that nodes often become hidden terminals preventing them from eavesdropping neighbors' traffic.

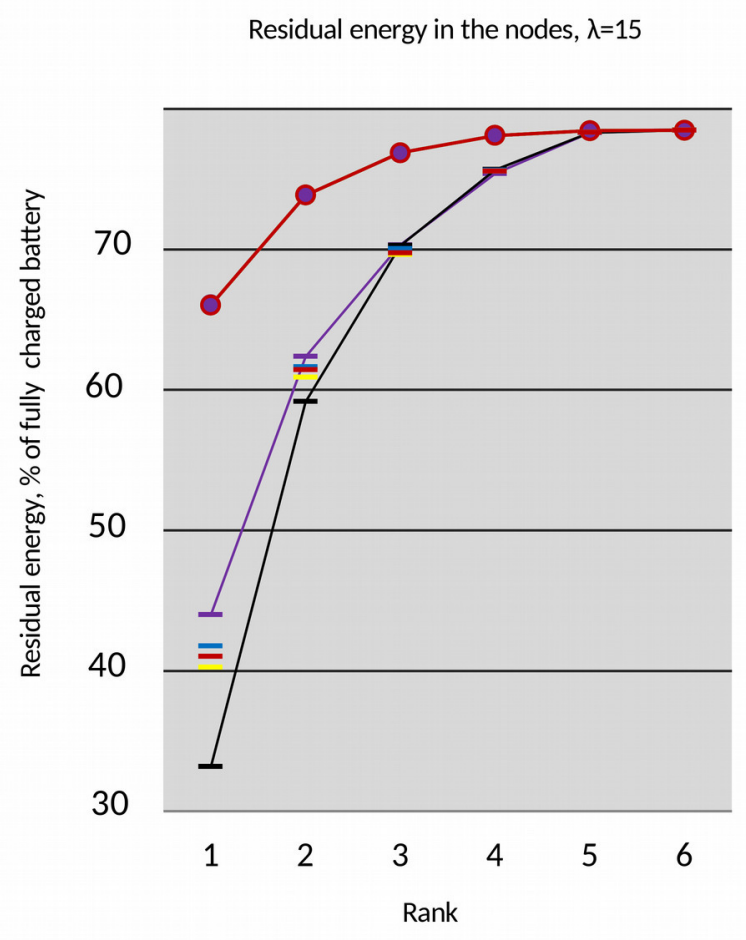

Fig 2. Residual-energy in the nodes after each node has transmitted 300 data packets
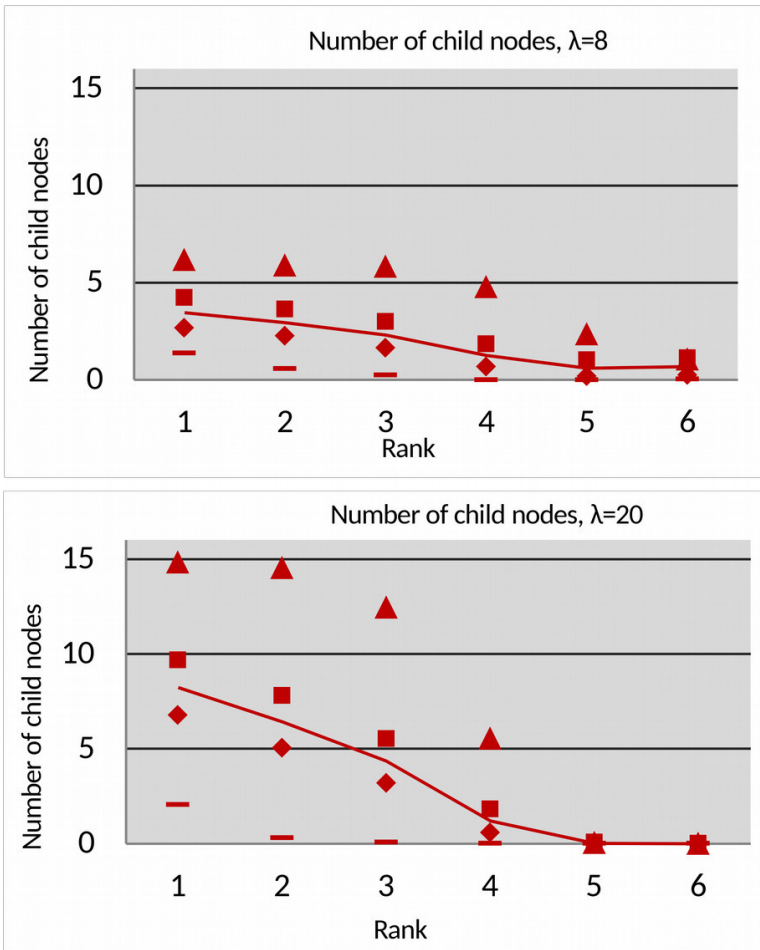

Fig 3. Number of child nodes versus rank for different node densities 
Our SPOF-algorithm described in subsection III.D and presented with purple line and marks in Figure 2, gives the best balancing effect. The reason is that higher-rank nodes are encouraged to choose paths that does not include the nodes that act as SPOF. However, SPOF nodes are not completely unloaded from forwarding data since child nodes have to forward all traffic through their SPOF parent nodes.

At higher rank nodes the SPOF-algorithm gives equal or marginally less balance compared to all other weighted-fair-sharing algorithms. However, the most efficient energy-balancing algorithm is the algorithm that focuses on energy balance among the lowest rank nodes, although this may give reduced balancing effect at the higher-rank nodes. The reason is that the lowest rank nodes always consumes the highest average amount of energy.

Increased number of child nodes enlarges energy imbalance between nodes, in particular for algorithms that uses fixed preferred-parent nodes. The number of child nodes increases with increased node density and reduced rank. This is demonstrated in Figure 3 which shows the number of child nodes versus rank for two different node densities, $\lambda=8$ and $\lambda=20$. This figure supports the findings in Figure 1 related to the rapidly increase in energy imbalance for increased node density. The circled shaped markers in Figure 3 with the corresponding lines show the average values. The square shaped and the diamond shaped markers shows the $95 \%$ confidence interval. In addition, the highest values, averaged over all different-seeds simulations are shown as triangular markers. The lowest values, averaged over all different-seeds simulations are shown as short horizontal lines.

\section{CONCLUSION}

Simulations presented in this paper show that the energy imbalance is substantial in network running the native RPL routing protocol. Thus, some kind of energy balancing algorithm should be used to prevent premature node depletion.

A total of nine energy balancing algorithms applicable for RPL running networks are analyzed in this paper. Six of the algorithms are based on various approaches suggested in literature. In addition, we suggest three new energy balancing approaches to complement the selection of algorithms. A common context is used to simulate and compare the performance of all the algorithms.

The simplest of our suggested approach is just a tiny adjustment of RPL's parent selection algorithm. Instead of using a preordain tiebreaker parameter, the preferred-parent (next-hop node) is randomly selected among the nodes in the parent-list. The adjustment gives a significant balancing effect. Especially in high density networks, where the residual-energy of the most depleted node is increased more than $10 \%$ compared to running native RPL.
The second and the third suggested algorithm use the nodes' residual-energy to weight-balance the transmitted traffic between all available parent nodes. In the second algorithm, a node with a single point of failure (SPOF) parent advertises a residual-energy level equal to the lowest of its own and its parent energy level. The algorithm requires residual-energy information to be exchanged between the nodes during RPL management packet exchange.

In the third algorithm, the nodes eavesdrop on the traffic in their vicinity to estimate neighboring nodes residual-energy level. Increased traffic density degrades the eavesdropping algorithm in the proximity of the sink.

Simulations shows that the SPOF algorithm performs best of all the weighting algorithms. Compared to native RPL is the SPOF algorithm increasing the residual-energy of the most depleted node with over $20 \%$.

However, the best energy balancing is achieved when nodes choose the preferred-parent as the member of the parents list that has the highest residual-energy level. Simulations of the algorithm show that, compared to native RPL, the residual-energy of the most depleted nodes increases by $25 \%$. The merit of this algorithm is that the lowest residual-energy paths are always avoided. On the contrary, weighting the traffic between all potential parent nodes means that also the lowest energy nodes are used, although rarely. However, to select the node with highest residual-energy, the nodes must exchange energy information. Randomly selecting the preferred-parent requires no extra information exchange.

Although the energy consumption is balanced, it is always the nodes closest to the sink that consumed the highest amount of energy. However, balancing the energy consumption of equal rank nodes can give reduced network management cost.

\section{REFERENCES}

[1] C. Buratti, A. Conti, D. Dardari and R. Verdone, «An Overview onWireless Sensor Networks Technology and Evolution,» Sensors, Volume 9 Issue 9, pp. 6869-6896, 2009. http://dx.doi.org/ 10.3390/s90906869

[2] Y. Liu, Y. He, M. Li, J. Wang, K. Liu and X. Li, «Does Wireless Sensor Network Scale? Measurement Study on GreenOrbs,» IEEE TRANSACTIONS ON PARALLEL AND DISTRIBUTED SYSTEMS, pp. 1983-1993, 2013. http://dx.doi.org/ 10.1109/TPDS.2012.216

[3] K. Heurtefeux, H. Menouar and N. AbuAli, «Experimental Evaluation of a Routing Protocol for WSNs: RPL robustness under study,» IEEE 9th International Conference on Wireless and Mobile Computing, Networking and Communications (WiMob), pp. 491-498, 2013. http://dx.doi.org/ 10.1109/WiMOB.2013.6673404

[4] T. W. e. al., «RPL: IPv6 Routing Protocol for Low-Power and Lossy Networks,» Request for Comments: 6550, 2012.

[5] P. L. e.al, «The Trickle Algorithm,» Request for Comments: 6206, 2011.

[6] P. O. Kamgueu, E. Nataf, T. D. Ndie and O. Festor, «Energy-based routing metric for RPL,» RR-8208, pp. 1-14, 2013.

[7] G. Xu and G. Lu, «Multipath Routing for DAG-based WSN with Mobile Sinks,» Proceedings of the 2nd International Conference on Computer Science and Electronics Engineering ICCSEE, pp. 1678$1682,2013$. 
[8] C. Abreu, M. Ricardo and P.M.Mendes, «Energy-aware routing for biomedical wireless sensor networks,» Journal of Network and Computer Applications, p. 270-278, 2014. http://dx.doi.org/ 10.1016/j.jnca.2013.09.015

[9] L. Chang, T. Lee, S. Chen and C. Liao, «Energy-Efficient Oriented Routing Algorithm in Wireless Sensor Networks,» International Conference on Systems, Man, and Cybernetics (SMC), pp. 3813 3818, 2013. http://dx.doi.org/ 10.1109/SMC.2013.651

[10] K. S. Shivaprakasha and M. Kulkarni, «Energy Efficient Shortest Path Routing Protocol for Wireless Sensor Networks,» International Conference on Computational Intelligence and Communication Networks CICN, pp. 333 - 337, 2011. http://dx.doi.org/ 10.1109/CICN.2011.70

[11] O.Iova, F. Theoleyre and T. Noel, «Improving the network lifetime with energy-balancing routing: Application to RPL,» Wireless and Mobile Networking Conference (WMNC), pp. 1 - 8, 2014. http://dx.doi.org/ 10.1109/WMNC.2014.6878864

[12] S. Chiang, C. Huang and K. C. Chang, «A Minimum Hop Routing Protocol for Home Security Systems Using Wireless Sensor Networks,» Transactions on Consumer Electronics, pp. 1483 - 1489, 2007. http://dx.doi.org/ 10.1109/TCE.2007.4429241

[13] P. T. A. Quang and D. Kim, «Enhancing Real-Time Delivery of Gradient Routing for Industrial Wireless Sensor Networks,» TRANSACTIONS ON INDUSTRIAL INFORMATICS, pp. 61-68, 2012. http://dx.doi.org/ 10.1109/TII.2011.2174249

[14] M. N. Moghadam, H. Taheri and M. Karrari, «Minimum cost load balanced multipath routing protocol for low power and lossy networks,» Wireless Networks, Volume 20, Issue 8, pp. 2469-2479, 2014. http://dx.doi.org/ 10.1007/s11276-014-0753-7

[15] R. Vidhyapriya and P. T. Vanathi, «Energy Efficient Adaptive Multipath Routing forWireless Sensor Networks,» IAENG International Journal of Computer Science, pp. 56-64, 2007.

[16] K. Sha, J. Gehlot and R. Greve, «Multipath Routing Techniques in Wireless Sensor Networks: A Survey,» Wireless Personal Communications, pp. 807-829, 2013. http://dx.doi.org/ 10.1007/s 11277-012-0723-2
[17] T. Rault, A. Bouabdallah and Y. Challal, «Energy efficiency in wireless sensor networks: A top-down survey,» Computer Networks, vol. Volume 67, p. 104-122, 2014. http://dx.doi.org/ 10.1016/j.comnet.2014.03.027

[18] Y. M. Lu and V. W. S. Wong, «An energy-efficient multipath routing protocol for wireless sensor networks,» International Journal of Communication Systems, p. 747-766, 2007. doi : 10.1002/dac.843

[19] A. Liu, J. Ren, X. Li, Z. Chen and X.Shen, «Design principles and improvement of cost function based energy aware routing algorithms for wireless sensor networks,» Computer Networks , p. 1951-1967, 2012. http://dx.doi.org/10.1016/j.comnet.2012.01.023

[20] N. A. Pantazis, S. A. Nikolidakis and D. D. Vergados, «EnergyEfficient Routing Protocols in Wireless Sensor Networks: A Survey,» Communications Surveys \& Tutorials, pp. 551-591, 2013. http://dx.doi.org/ 10.1109/SURV.2012.062612.00084

[21] C. Efthymiou, S. Nikoletseas and J. Rolim, «Energy balanced data propagation in wireless sensor networks,» Journal Wireless Networks , p. 691-707, 2006. http://dx.doi.org/ 10.1007/s11276-006-6529-y

[22] C. M. Angelopoulos, G. Filios, S. Nikoletseas, D. Patroumpa, T. P. Raptis and K. Veroutis, «A Holistic IPv6 Test-Bed for Smart, Green Buildings,» International Conference on Communications (ICC), pp. 6050-6054, 2013. http://dx.doi.org/ 10.1109/ICC.2013.6655569

[23] «OMNET++ : http://www.omnetpp.org/. (Mars 2015)».

[24] A.-L. Kampen, K. Øvsthus, L. Landmark and Ø. Kure, «Energy Reduction in Wireless Sensor Networks by Switching Nodes to Sleep During Packet Forwarding,» The Sixth International Conference on Sensor Technologies and Applications, SENSORCOMM, pp. 189$195,2012$.

[25] C. R. F. H. IEEE P802.15 Working Group, «Part 15.4: Wireless Medium Access Control (MAC) and Physical Layer (PHY) Specifications for Low-Rate Wireless Specifications for Low-Rate Wireless,» IEEE Std 802.15.4 ${ }^{\mathrm{TM}}$-2006, 2006.

[26] K. K. Gagneja and K. E. Nygard «A QoS based Heuristics for Clustering inTwo-Tier Sensor Networks,» Proceedings of the Federated Conference on Computer Science and Information Systems, FedCSIS, pp. 779-784, 2012. 